\author{
Marco AP Safadi ${ }^{1,2}$, E David G Mcintosh ${ }^{3,4}$ \\ 1 Department of Pediatrics, Santa Casa de Sao Paulo School of Medicine, Sao Paulo, Brazil \\ 2 Pediatric Infectious Diseases Division, Sao Luiz Hospital, Sao Paulo, Brazil \\ ${ }^{3}$ Novartis Vaccines and Diagnostics, Amsterdam, The Netherlands \\ ${ }^{4}$ Imperial College, London, UK
}

\title{
Epidemiology and prevention of meningococcal disease: a critical appraisal of vaccine policies
}

Author for correspondence:

e-mail: masafadi@uol.com.br

Статья поступила: 10.11.2011 г., принята к печати: 15.01.2012 г.

Meningococcal disease is characterized by a marked variation in incidence and serogroup distribution by region and over time. In several European countries, Canada and Australia, immunization programs, including universal vaccination of infants or toddlers with catch-up campaigns in children and adolescents, aimed at controlling disease caused by meningococcal serogroup $C$ have been successful in reducing disease incidence through direct and indirect protection. More recently, meningococcal conjugate vaccines targeting disease caused by serogroups $A, C, W$ - 135 and $Y$ have been licensed and are being used in adolescent programs in the USA and Canada while a mass immunization campaign against serogroup $A$ disease has been implemented in Africa. Positive results from clinical trials using vaccines against serogroup $B$ disease in various age groups suggest the possibility of providing broader protection against serogroup $B$ disease than is provided by the currently used outer membrane vesicle vaccines. The purpose of our review of meningococcal epidemiology and assessment of existing policies is to set the stage for future policy decisions. Vaccination policies to prevent meningococcal disease in different regions of the world should be based on quality information from enhanced surveillance systems.

Key words: herd protection, immunization, meningococcal disease, meningococcal vaccine, Neisseria meningitidis, public health.

Meningococcal disease is characterized by a marked variation in incidence and serogroup distribution by region and over time. In several European countries, Canada and Australia, immunization programs, including universal vaccination of infants or toddlers with catchup campaigns in children and adolescents, aimed at controlling disease caused by meningococcal serogroup $C$ have been successful in reducing disease incidence through direct and indirect protection. More recently, meningococcal conjugate vaccines targeting disease caused by serogroups A, C, W-135 and $\mathrm{Y}$ have been licensed and are being used in adolescent programs in the USA and Canada while a mass immunization campaign against serogroup $A$ disease has been implemented in Africa. Positive results from clinical trials using vaccines against serogroup $B$ disease in various age groups suggest the possibility of providing broader protection against serogroup B disease than is provided by the currently used outer membrane vesicle vaccines. The purpose of our review of meningococcal epidemiology and assessment of existing policies is to set the stage for future policy decisions. Vaccination policies to prevent meningococcal disease in different regions of the world should be based on quality information from enhanced surveillance systems.

Neisseria meningitidis has become a leading cause of bacterial meningitis and sepsis in infants, children, adolescents and young adults. This has become more noticeable after the introduction of Haemophilus influenzae type $b$ and pneumococcal conjugate routine vaccination in manycountries, which has resulted in significant reductionsin those infections. Globally, up to 500,000 cases of invasive meningococcal disease occur annually, resulting in an estimated 50,000 deaths [I]. Invasive meningococcal disease is often sudden in onset, rapidly progressive and potentially fatal in outcome [2]. The increasing use of PCR for diagnosis has increased case detection by up to $30 \%$ [3]. Survivors are liable to have long-term consequences such as cognitive impairment, deafness and the need for amputation [4].

Serogroups A, B, C, W-135, Y and X are responsible for virtually all cases of invasive meningococcal disease. Meningococci have been shown to have the potential capacity to exchange the genetic material responsible for capsular production and to switch capsular phenotype. Antigenic variations of capsular and surface proteins resulting from these genetic mechanisms enhance the ability of meningococci to evade immune responses. This partly explains the organism's capability of causing outbreaks of meningococcal disease. The epidemiology of meningococcal disease varies markedly by region. In Europe and in the Americas, the disease is mostly endemic, with average incidence rates ranging from less than 0,5 to 8,9 cases per 100,000 total population $[5,101,102]$. 
After the introduction of meningococcal $\mathrm{C}$ conjugate vaccines into the routine immunization programs of several countries in Europe, serogroup $C$ disease has declined dramatically, an occurance unlikely to be due to natural variation - leaving these countries with a predominance of serogroup $B$ disease [6]. In the USA, serogroups $B, C$ and $Y$ each cause approximately a third of cases [7]. Serogroups $B$ and $C$ are responsible for the majority of cases in Latin America, although there are increasing reports of sero-group $\mathrm{W}$-135 (including in Florida, USA, which has a large Latin American population) and serogroup $Y$ disease $[5,8]$.

The 'meningitis belt' in Africa reports the highest incidence rates of disease in the world and is primarily affected by serogroup A [9, 10], although other serogroups such as W-135 in Burkina Faso $[11,12]$ and $X$ in western Kenya $[13,14]$, Burkina Faso, Togo [15] and Niger [16] are emerging in this region. By contrast to disease in the developed world, which is commonly sporadic, there is seasonality of disease in this region with recurrent epidemics [9].

Surveillance data on meningococcal disease epidemiology in Asia and the Middle East are scant and limited, with low incidence rates of disease reported and infrequent outbreaks or epidemics [17]. For example, one survey performed in the city of Hefei in China reported an annual incidence of between 1,19 and 2,86 per 100,000 total population between 2003 and 2007 [18]. There was an upwards shift in age distribution over this period towards those aged 12-17 years, with an incidence of 6,57 per 100,000 inhabitants. All isolates that were serogrouped were found to be serogroup $C$. There are Chinese reports of outbreak control using plain polysaccharide vaccine. Serogroup $A$ is common in many parts of Asia, but cases of serogroups $B$ and $C$ disease are increasingly reported in the region [17]. A serogroup A outbreak occurred in and around New Delhi in 2005-2006, during which there was effective use of not only isolation, antibiotic chemotherapy and chemoprophylaxis, but also of immunization [19, 20]. Outbreaks of meningococcal disease caused by the W-135 serogroup have been reported among nomadic Muslims in Saudi Arabia. Vaccination using the serogroup A vaccine has been successful in decreasing infection associated with the Hajj [21].

The introduction of meningococcal $\mathrm{C}$ conjugate vaccines into national immunization programs in several European countries, Canada and Australia resulted in a dramatic and immediate reduction in the incidence of meningococcal disease caused by serogroup $C$. Three monovalent serogroup $C$ conjugate vaccines are currently licensed. Two vaccines are based on oligosaccharides derived from serogroup C capsular polysaccharide conjugated to the nontoxic CRM197 mutant diphtheria toxin (Menjugate by Novartis Vaccines and Diagnostics, Siena, Italy, and Meningitec by Wyeth [now Pfizer] Vaccines, NY, USA). The other vaccine is based on a de-O-acetylated oligosaccharide conjugated to tetanus toxoid (Neisvac-C by Baxter Vaccines, Beltsville, MD, USA). There is no evidence to favor one vaccine over another in the routine clinical setting. A striking feature of these meningococcal $\mathrm{C}$ conjugate vaccination programs, which included catch-up of children and adolescents using different immunization schedules, has been the additional decrease in disease incidence in unvaccinated individuals as a result of herd protection [6]. Immunization of adolescents and young adults (the age groups that have the highest rates of colonization) in these catch-up campaigns reduced the carriage rates of meningococcal serogroup $\mathrm{C}$ and may have prevented transmission of the organism and acquisition by other individuals.

One of the key lessons learned with the meningococcal serogroup $C$ vaccination programs was the importance of herd protection to their success. However, the duration of individual immunity and of herd protection is as yet unknown, and surveillance of disease is crucial to address all of these issues. There is a focus on the pros and cons of infant versus single-dose toddler routine schedules after mass immunization programs [22]. After a successful mass immunization campaign, the institution of a singledose toddler schedule has merits and 'clears' the infant schedule in readiness for new vaccines. However, in countries with a high burden of disease in infants, this cost-effective approach has yet to prove its feasibility. In the UK there is discussion about removing one of the infant doses. Recent data showing waning immunity after meningococcal $C$ conjugate vaccination suggest the need for a booster dose during adolescence to maintain sustained population immunity against the disease [23-25].

In health systems where meningococcal $\mathrm{C}$ conjugate vaccines have been extensively deployed (TABLE) [26-35, 103], there is now increasing interest in the control of other serogroups, such as, serogroups A, B, W-135 and $Y$ The introduction of multivalent vaccines such as the conjugate vaccine against serogroups A, C, W-135 and $Y$ has the potential to reduce further the incidence of infection [36-38]. Most countries where meningococcal conjugate vaccines are licensed have a policy for vaccinating at-risk individuals.

The global incidence of serogroup $B$ infection is estimated to be between 20,000 and 80,000 cases per year, with a $10 \%$ fatality rate [104]. There is a need for a vaccine against

Table. Countries with recommendations for the use of meningococcal serogroup $\mathrm{C}$ conjugate vaccines

\begin{tabular}{|c|c|c|c|c|c|c|c|}
\hline \multirow[b]{2}{*}{ Country } & \multirow[b]{2}{*}{ Region } & \multicolumn{2}{|c|}{ Routine immunization } & \multirow[b]{2}{*}{ Booster } & \multicolumn{2}{|c|}{ Catch-up program } & \multirow[b]{2}{*}{ References } \\
\hline & & Ages & $\begin{array}{c}\text { Year } \\
\text { implemented }\end{array}$ & & Ages & $\begin{array}{c}\text { Year } \\
\text { implemented }\end{array}$ & \\
\hline \multicolumn{2}{|l|}{ Australia } & 12 months & 2003 & No & $1-19$ years & 2003 & 26 \\
\hline \multirow[t]{2}{*}{ Belgium } & $\begin{array}{l}\text { Wallonia and } \\
\text { Brussels }\end{array}$ & 12 months & 2002 & No & $1-5$ years & 2002 & \multirow[t]{2}{*}{27} \\
\hline & Flanders & 12 months & 2005 & No & $1-17$ years & 2001 & \\
\hline Brazil & - & $<2$ years & 2010 & $12-15$ months & None & & 5 \\
\hline
\end{tabular}


Table. Countries with recommendations for the use of meningococcal serogroup C conjugate vaccines (continue)

\begin{tabular}{|c|c|c|c|c|c|c|c|}
\hline \multirow[b]{2}{*}{ Country } & \multirow[b]{2}{*}{ Region } & \multicolumn{2}{|c|}{ Routine immunization } & \multirow[b]{2}{*}{ Booster } & \multicolumn{2}{|c|}{ Catch-up program } & \multirow[b]{2}{*}{ References } \\
\hline & & Ages & $\begin{array}{c}\text { Year } \\
\text { implemented }\end{array}$ & & Ages & $\begin{array}{c}\text { Year } \\
\text { implemented }\end{array}$ & \\
\hline \multirow{5}{*}{ Canada } & $\begin{array}{l}\text { Quebec } \\
\text { and Alberta }\end{array}$ & 12 months & 2002 & 12 years & $\begin{array}{c}2 \text { months - } \\
20 \text { years }\end{array}$ & $\begin{array}{c}2001 \\
\text { (Quebec) }\end{array}$ & \multirow{5}{*}{28} \\
\hline & $\begin{array}{l}\text { British } \\
\text { Columbia }\end{array}$ & $\begin{array}{l}2 \text { months, } \\
12 \text { months }\end{array}$ & 2003 & 12 years & $9-18$ years & 2001 onward & \\
\hline & $\begin{array}{l}\text { Prince Edward } \\
\text { Island }\end{array}$ & 12 months & 2003 & 12 years & - & - & \\
\hline & $\begin{array}{l}\text { Canada except } \\
\text { for Manitoba }\end{array}$ & 12 months & 2005 & 12 years & - & - & \\
\hline & Manitoba & $10-11$ years & 2005 & - & - & - & \\
\hline \multicolumn{2}{|l|}{ France } & $1-2$ years & 2009 & No & $1-24$ years & 2009 & 29 \\
\hline \multicolumn{2}{|l|}{ Germany } & $11-23$ months & 2006 & No & & & \\
\hline \multicolumn{2}{|l|}{ Greece } & 2 months, 4 months & 2006 & $15-18$ months & $0-6$ years & 2001 & 30 \\
\hline \multicolumn{2}{|l|}{ Iceland } & 6 months, 8 months & 2002 & No & & & 27 \\
\hline \multicolumn{2}{|l|}{ Ireland } & 4 months, 6 months & 2000 & 13 months & $0-22$ years & 2000 & 27 \\
\hline \multicolumn{2}{|l|}{ Italy } & $\begin{array}{c}2 \text { months - } \\
2 \text { years }\end{array}$ & 2005 & & & & 103 \\
\hline \multicolumn{2}{|c|}{ Luxembourg } & 13 months & 2001 & No & $1-19$ years & 2001 & 27 \\
\hline \multicolumn{2}{|c|}{ The Netherlands } & 14 months & 2002 & No & $\begin{array}{c}14 \text { months - } \\
18 \text { years }\end{array}$ & 2002 & 31,32 \\
\hline \multicolumn{2}{|l|}{ Portugal } & 3 months, 5 months & 2006 & 15 months & & & 27 \\
\hline \multirow[t]{2}{*}{ Spain } & $\begin{array}{l}\text { Most regions } \\
\text { (16 out of } 19)\end{array}$ & & & & $<6$ years & 2000 & \multirow[t]{2}{*}{33} \\
\hline & $\begin{array}{l}\text { Some regions } \\
\text { (three out of } 19 \text { ) }\end{array}$ & & & & Adolescence & 2000 & \\
\hline \multicolumn{2}{|c|}{ Switzerland } & $12-15$ months & 2006 & $11-15$ years & & & 27 \\
\hline \multirow{2}{*}{\multicolumn{2}{|c|}{ USA }} & \multirow{2}{*}{$11-12$ years } & 2005 & & & & \multirow{2}{*}{34} \\
\hline & & & 2010 & $16-18$ years & & & \\
\hline UK & & 3 months, 4 months & 1999 & 12-13 months & $\begin{array}{c}2 \text { months - } \\
18 \text { years }\end{array}$ & 1999 & 35 \\
\hline
\end{tabular}

serogroup B disease, the licensing and deployment of which has the potential to address this elusive serogroup [8].

In countries with meningococcal vaccination policies, establishment of enhanced surveillance systems is crucial in order to monitor the impact of meningococcal vaccination programs on the epidemiology of meningococcal disease. Given the potential impact of meningococcal disease on individuals and communities, a critical appraisal of the different policies used by various countries is insightful, and such an appraisal is the purpose of this article.

In order to do this, our starting point was to describe the policies used in countries with universal indications for meningococcal serogroup $\mathrm{C}$ conjugate and quadrivalent vaccines, to move to policies for outbreak control and travel, and then to give a short country/regional overview. It is sometimes necessary to deduce policy decisions from how meningococcal vaccines are used in different situations. Underlying this approach was a description of epidemiology and changing epidemiology.

\section{Prevention of meningococcal infections in different healthcare systems}

The licensing and widespread deployment of vaccines against $N$. meningitidis serogroup $C$ in several countries has resulted in substantial reductions in infections caused by this organism.

\section{United Kingdom}

Since the introduction of universal vaccination against N. meningitidis serogroup C in 1999, brought about by the emergence of cases of invasive meningococcal disease due to sequence type 11 (ST-11) clonal complex [39], the number of reported cases per year has decreased dramatically, from 955 in 1998/1999 to 13 in 2008/2009 (FIGURE I) [105].

In fact, the UK was the first country worldwide to implement a national meningococcal $C$ conjugate vaccination program [35]. The vaccine was introduced into the infant schedule at 2, 3 and 4 months of age along with a catchup program for those aged up to 18 (later extended to 24) 
Figure I. Meningococcal Reference Unit isolates of Neisseria meningitidis: England and Wales by serogroup and epidemiological year 1998/1999-2008/2009.

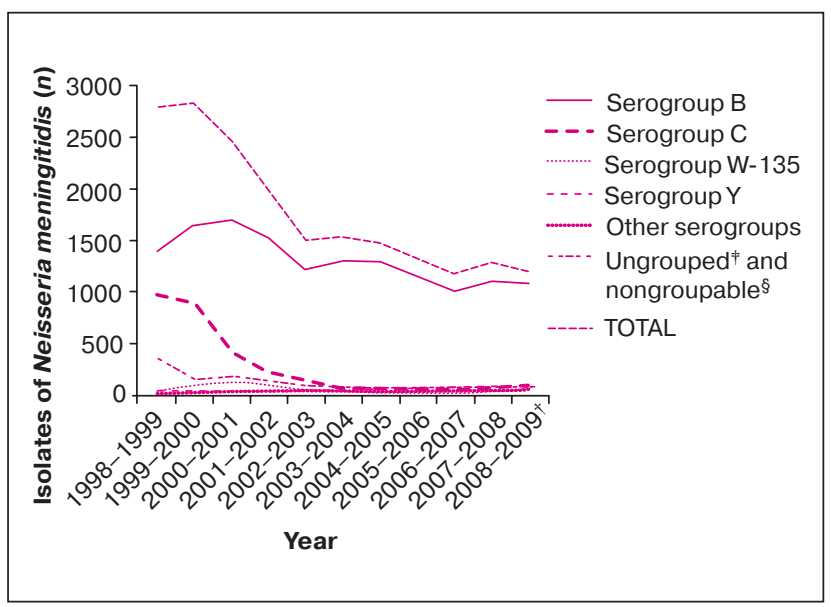

Примечание. ${ }^{\dagger}$ Provisional data. ${ }^{\dagger}$ This term is used for PCR reports where meningococcal DNA has been detected but further testing to determine the serogroup has not been successful.

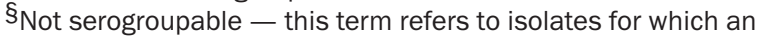
expressed capsule was unable to be determined. These reports are unlikely as, by definition, organisms from sterile sites are generally expressing capsules. This result is often associated with throat swab or nose swab isolates. Data taken from [105].

years. During the first year of the program, the effectiveness was approximately $87 \%$; over a longer time period, the effectiveness remained high in those who had been vaccinated as part of the catch-up program [40, 41]. However, waning efficacy was reported among children immunized during their first year of life [40], leading to changes in the immunization schedule to two doses at 3 and 4 months of age, followed by a booster dose administered at 12 months of age, in order to confer longer lasting protection.

Recently, postlicensure data showed that only $12 \%$ of those aged $<6$ months who had been vaccinated had serum bactericidal antibody titers $>1: 8$ (the serologic surrogate of protection being 1:8) by year 6 postvaccination, with an apparent nadir of bactericidal antibody decline reached within 4 years [25]. Despite this decline in antibody persistence in the infant cohorts, there has been a sustained decrease in invasive meningococcal $\mathrm{C}$ disease, the incidence now standing at 0.02 per 100,000 total population [41]. A degree of herd protection in England and Wales was also observed $[6,28]$, which could be related to the sca.clscic3.lly significant effect of the vaccine on nasopharyngeal carriage [42].

\section{The Netherlands}

Since 2002, another country that has had a successful program of immunization against meningococcal serogroup $C$ disease is The Netherlands (FIGURE 2) [106]. Because of increasing serogroup $C$ meningococcal disease in 2000 to 2001, a vaccination program using meningococcal C conjugate vaccine was instituted in 2002 [31]. In contrast to the UK, The Netherlands chose to immunize toddlers with one dose at 14 months of age followed by a catchup campaign including all children and adolescents between 1 and 18 years of age, which led to an almost complete disappearance of serogroup $\mathrm{C}$ disease [31, 32].
Figure II. Meningococcal disease in The Netherlands.

(A) Number of patients with serogroup $C$ invasive meningococcal disease by age group and year in The Netherlands, 2001-2008. (B) Number of patients with serogroup B invasive meningococcal disease by age group and year in The Netherlands, 2001-2008, Data taken from [106].

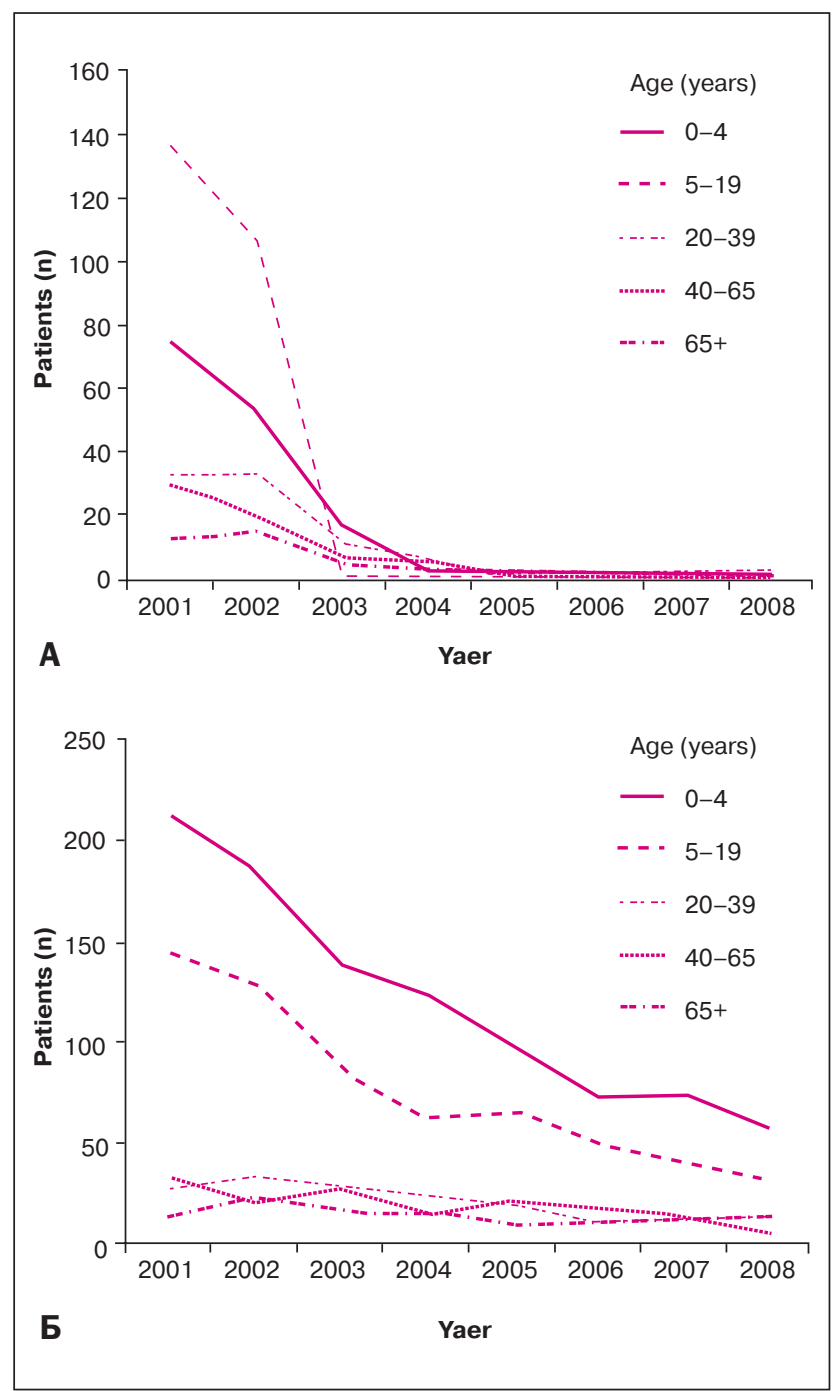

By 2006 there were only four reported cases of serogroup $C$ disease [106]. Herd protection may also have contributed to the decrease in serogroup $\mathrm{C}$ meningococcal disease. The Dutch strategy requires that those not receiving the meningococcal $\mathrm{C}$ conjugate vaccine, for example those less than 14 months of age, be closely monitored because they do not have protective serum bactericidal antibody titers [32]. A rapid waning of protective vaccine-induced antibodies was also seen in the Dutch population within a few years of vaccination, especially in those immunized before 6 years of age. Therefore, administration of a booster dose early in adolescence is being considered to provide protection during this period of increased risk of meningococcal disease [43].

\section{Greece}

It has been noted that although the meningococcal C conjugate vaccine had not been introduced into the official national immunization schedule, Greek pediatricians had administered the vaccine to nearly $100 \%$ of infants and children 2-24 months of age since its introduction in 
Greece in January 2001, with $72 \%$ of pediatricians having administered it as a single dose to children $>12$ months of age by 2006 [30]. By 2004, the number of pediat-ric cases of invasive meningococcal serogroup $C$ disease in those $<15$ years of age had decreased from approximately 50 cases per year in the late 1990s to zero, although there were subsequently three cases in 2005 and four in 2006. By 2004, serogroups B and A were predominating [30]. In April 2011, quadrivalent meningococcal conjugate vaccine was recommended by the Greek government to replace monovalent serogroup $C$ conjugate vaccine in Greece in the national schedule, along with an adolescent booster, although reimbursement is pending.

\section{Spain}

Meningococcal $\mathrm{C}$ conjugate vaccine was introduced in Spain in late 2000 , generally with a 2-, 4- and 6-month schedule along with a catch-up program in most regions of the country [33]. The catch-up initially targeted those $<6$ years of age but subsequently extended to 18 years. Coverage for both the infant schedule and the catchup program was reported to be between 90 and $95 \%$. For infants and children up to 9 years of age, the rate of serogroup $C$ disease decreased from 7,04 per 100,000 in the prevaccination period from 1999 to 2000 , to 1,08 per 100,000 in the postvaccination period. The overall vaccine effectiveness was reported to be 95,2\% (95\% Cl: 91,2-97,3\%) for the infant schedule and 97,8\% (95\% Cl: 98,1-99,9\%) for the catch-up. Herd protection effects in Spain were less pronounced than in the UK or The Netherlands, probably because the catchup campaign in Spain initially targeted only children aged $<6$ years, not including teenagers, the age group where carriage rates are highest.

\section{France}

Peaks of serogroup $C$ infection had been observed in France in 1992 and 2003, the isolates responsible being from ST-11 clonal complex [44]. The same clonal complex with a new phenotype has been occurring in clusters in France. France was without a routine immunization program against serogroup C infection until 2009 when the emergence of the new virulent clones of $N$. meningitidis serogroup $C$ posed a new threat. In 2009 , meningococcal $C$ conjugate vaccine was recommended by the national authorities for toddlers between 1 and 2 years of age, along with a catch-up for children, adolescents and adults up to 24 years of age [29].

Another notable policy decision in France was to use a meningococcal serogroup B vaccine, designed 20 years previously for Norway, to control a clonal outbreak of serogroup B disease in Normandy [45]. The decision was vindicated with the reduction in target cases in the target population (all inhabitants younger than 20 years of age living in Normandy) from 31,6 to 5,9 per 100,000 .

\section{Brazil}

During the 1970s, Brazil suffered its largest recorded epidemic of meningococcal disease, with its epicenter in Sao Paulo, and was characterized by two overlapping epidemic waves, the first provoked by serogroup C meningococcus, starting in April 1971, and the second provoked by serogroup A meningococcus, beginning in April
1974. The incidence rate reached a peak of 179 cases per 100,000 total inhabitants in 1974. This epidemic provided the first major experience in the world with polysaccharide $A$ and $C$ vaccines on a large scale, resulting in control of the epidemic from 1975 [46].

From 2002 onward, a significant increase in the proportion of cases attributed to serogroup C associated to the ST-103 complex was observed; currently, serogroup $\mathrm{C}$ is the most frequent serogroup causing meningococcal disease in Brazil. Emergence of serogroup W-135, associated to the ST-11 complex, was recently reported in the states of Sao Paulo, Rio de Janeiro and Rio Grande do Sul. Several outbreaks of meningococcal disease caused by serogroup $C$ were reported in Brazil in recent years, affecting different cities. As a strategy to control these outbreaks, besides chemoprophylaxis to contacts, vaccination is recommended to the population age groups with higher risk. In these programs, meningococcal $\mathrm{C}$ conjugate vaccines are used for children less than 2 years of age and meningococcal $A / C$ polysaccharide vaccines are used for those 2 years of age and above [5].

The meningococcal C conjugate vaccine is also funded by the government and recommended for selected high-risk groups (patients with asplenia, individuals with storage diseases, children and adolescents up to 13 years of age infected with HIV, individuals with humoral immunodeficiency and candidates for cochlear implant). Such policies for vaccinating those at high risk for invasive meningococcal infection are practised in many countries where meningococcal vaccines are licensed, in a similar way to recommendations published by the US CDC [34]. In 2010, Brazil was the first Latin-American country to incorporate the meningococcal $C$ conjugate vaccine in the routine immunization program, with two doses in the first year, at 3 and 5 months of age, and a booster dose at 12-15 months [107]. Toddlers between 12 and 23 months of age receive one dose of the vaccine. Currently, no catchup campaign for older age groups is planned.

\section{Australia}

In 1987, there was an outbreak of serogroup A meningococcal disease in the Aboriginal population, which was followed by an increase in disease caused by serogroups $B$ and $C$ nationwide [47]. The meningococcal $C$ conjugate vaccine was introduced into the Australian routine immunization schedule in 2003 with a single dose at 12 months of age, along with a catch-up for individuals less than 20 years of age [48]. This resulted in a 75\% reduction in nationally notified laboratory-confirmed cases from 213 in 2002 to 50 in 2005 . Also, overall meningococcal mortality rates fell from a peak of 0,24 per 100,000 people in 2002 to 0,12,0.11, 0,09 and 0,06 per 100,000 in 2003, 2004, 2005 and 2006, respectively [48]. By 2009, 83\% of laboratory-confirmed cases where a serogroup was determined arose from serogroup B [49].

\section{Canada}

There was a staggered introduction of meningococcal C conjugate vaccine in Canada as shown in TABLE [28]. The average incidence of invasive meningococcal disease in the population overall decreased from 0,62 per 100,000 in 2002 to 0.42 per 100,000 in 2006. The incidence of serogroup $C$ disease decreased significantly from 0,23 per 
100,000 in 2002 to 0,08 per 100,000 in 2006, whereas that of serogroups $B, Y$ and $W-135$ remained stable. $A$ decrease in serogroup $C$ disease in adults 30 years of age and older in Canadian provinces with early programs suggested the possibility of herd protection. There was no evidence of serogroup replacement. In Quebec, the incidence of serogroup $C$ disease decreased from a peak of 1.4 per 100,000 to $<0,1$ per 100,000 total population over a 7 -year follow-up period from 2001 [50]. Again, there was evidence of herd protection.

In 2009, the National AdvisoryCommittee on Immunization recommended immunization with the quadrivalent meningococcal conjugate vaccine in early adolescence, even if meningococcal conjugate vaccine had been received as part of a routine 1-year-old infant vaccination program. In some provinces, the recommendation for the toddler dose of meningococcal $\mathrm{C}$ conjugate vaccine was changed to the quadrivalent conjugate vaccine.

\section{United States}

The Advisory Committee on Immunization Practices of the USA updated meningococcal immunization recommendations in October 2010 [34]. The previous recommendations from 2005 had been routine vaccination of young adolescents (persons aged 11-12 years) with quadrivalent meningococcal conjugate vaccine at the preadolescent healthcare visit. However, because of declining immunity at 5 years postvaccination, a booster dose given 3-5 years after initial immunization was recommended in October 2010. Furthermore, a recommendation for a two-dose schedule (with doses 2 months apart) for those 2-54 years of age with certain underlying conditions has also been introduced. These vaccinees were also recommended to receive a booster dose after 5 years. Serogroup CY-Hib conjugate vaccine as well as MenACWY-CRM vaccine may become available in the USA for infants and toddlers [51].

\section{Cuba}

An outbreak of serogroup B meningococcal disease was reported in 1983 with an incidence of 14.4 per 100,000 total inhabitants [52]. Beginning in 1982, a vaccination program was initiated in high-risk groups and subsequently in infants, children and young adults 3 months to 24 years of age using a locally produced serogroup B outer membrane vesicle vaccine (OMV) plus a serogroup C polysaccharide vaccine. Since 1991, the vaccine has been routinely used in their childhood immunization schedule at 3 and 5 months of age, resulting in a decrease in disease incidence rates of invasive meningococcal disease from 6,5 cases per 100,000 population per year in 1989 to 0,8 per 100,000 population per year in 1993 [52-54].

\section{African meningitis belt countries}

Epidemic and endemic rates of meningococcal disease in sub-Saharan Africa remains a serious public health concern. Control of these epidemics associated to $N$. meningitidis serogroup $A$ has relied on a reactive vaccination strategy with polysaccharide vaccines that demonstrated limitations in interrupting these epidemics [55]. The Meningitis Vaccine Project, a partnership between the WHO and PATH with funding from the Bill and Melinda Gates Foundation, has developed a meningococcal A conjugate vaccine, manufactured at the Serum Institute of India (called MenAfriVac ${ }^{\mathrm{TM}}$ ) and prequalified by the WHO in 2010. Clinical trials in Africans 1-29 years of age demonstrated that the vaccine elicited a significantly stronger and persistent response to group $A$ antibody than the quadrivalent polysaccharide vaccine, with similar reactogenicity profile. The vaccine also induced immunologic memory [56]. Introduction and widespread use of this vaccine targeting children, adolescents and adults 1-29 years of age in subSaharan countries is expected to generate herd immunity, providing a dramatic impact on disease incidence and carriage, controlling serogroup A disease not only in the vaccinated cohorts but also in infants and adults more than 30 years of age.

\section{Systems using vaccines for outbreak control}

Meningococcal polysaccharide vaccines have played a pivotal role in disease prevention for several decades. They proved to be safe and effective in controlling outbreaks and epidemics. However, in common with other unconjugated polysaccharide vaccines, they do not generate an adequate immune response in young children. Even in patients over 2 years of age, the protection offered is of limited duration. Furthermore, they are capable of inducing hypo-responsiveness after subsequent doses [1, 46].

Meningococcal polysaccharide-protein conjugate vaccines represent a major advance in meningococcal disease prevention. The conjugation of polysaccharides to protein carriers (nontoxic diphtheria mutant toxin [CRM197] or tetanus toxoid) alters the nature of the antipolysaccharide response to a T-cell-dependent response. When $B$ cells recognize the polysaccharide, they process the conjugated carrier protein and present peptide epitopes to $\mathrm{CD} 4^{+} \mathrm{T}$ cells. This antigenic complex induces the production of elevated antibody levels, including in young infants, higher antibody avidity and increases serum bactericidal activity. Unlike unconjugated polysaccharide vaccines, they can induce immuno-logic memory providing an excellent amnestic response (booster effect) on re-exposure. They can also reduce nasopharyngeal carriage of Neisseria meningitidis and so unvaccinated individuals are protected indirectly through a herd immunity effect $[1,46]$.

In the 1990s, France, the UK, The Netherlands, Switzerland, Spain and many African countries used a bivalent, plain polysaccharide, serogroup $A$ and $C$ vaccine for outbreak control, whereas the USA, Canada, Italy, Portugal, Denmark and Saudi Arabia routinely used a quadrivalent plain polysaccharide $A, C, W-135$ and $Y$ vaccine [57]. This approach brings about only temporary benefits, and a number of countries, including Canada [Ю8], now suggest that conjugate vaccines are preferable for dealing with outbreaks. In a survey of European countries in 2007 administered by the European Union Invasive Bacterial Infections Surveillance Network, $74 \%(n=17)$ of respondents recommended meningococcal vaccination in addition to chemo-prophylaxis for close contacts in an outbreak situation, if illness in the outbreak is due to a vaccine-preventable serogroup [58]. Furthermore, nine countries had defined epidemiologic thresholds to commence local or regional vaccination campaigns during outbreaks of meningococcal infection. Arising from this was the recommendation for 'the development of guidance 
for best practice in priority areas, based on evidence or consensus' [58]. This guidance, published in 2010, states, 'If a case of meningococcal disease is caused by a strain that is preventable by an available licensed vaccine, vaccination in addition to chemoprophylaxis should be offered to household contacts unless considered to be already immune' [109].

The examples par excellence of an outbreak approach have been the meningococcal immunization campaigns in New Zealand. A meningococcal serogroup A outbreak occurred in Auckland, New Zealand, in 1985 to 1986, with incidence rates reaching 8.3 per 100,000 total inhabitants, and led to a vaccination campaign with meningococcal serogroup A polysaccharide vaccine [59]. Infants and children 3 months to 19 years of age were targeted, particularly Maori and Pacific Islander populations. The campaign was considered a success because no cases of serogroup A disease were reported in children during the program [59].

An epidemic of serogroup B disease began in 1991 in New Zealand, with incidence rates reaching 17,4 per 100,000 total inhabitants in 2003 [60]. ST-41/44 clonal complex accounted for approximately $85 \%$ of the cases [60]. A tailor-made, strain-specific serogroup $B$ vaccine using the OMVs from ST-41/44 clonal complex and known as MeNZB (Norwegian Institute of Public Health and Novartis Vaccines) was introduced in 2004 and was reported to be $80 \%$ effective in infants and children $<5$ years of age [61] in the first year after immunization. However, in the second year after immunization the effectiveness was significantly lower, coinciding with the decline in the proportion of children who maintained serum bactericidal antibodies in protective titers $[62,63]$. Further analysis suggests that, for success, not only high antibody titers following the priming course are necessary but also boosting is a prerequisite for longer-term protection [64]. This meningococcal immunization program was terminated in 2006.

In Norway, an outbreak of serogroup B disease associated with ST-32 clonal complex started in 1974 [65]. Such strain-specific vaccines were instrumental in controlling outbreaks in both Norway and Normandy [66, 67]. As mentioned previously in the section on France, in the target population in Normandy (all inhabitants younger than 20 years of age living in Normandy), there was a decrease from 31,6 per 100,000 to 5,9 per 100,000 in invasive meningococcal infection between 2006 and 2009 [52].

Owing to the fact that antibiotics are often used in outbreak control and notwithstanding the fact that meningococcal resistance is relatively low [68], the use of meningococcal vaccine in outbreak control does have the potential to limit antibiotic usage by virtue of preventing outbreaks from spreading.

While economic data on the cost of outbreak control are scant, a 1998 effort in the USA was documented after seven patients aged 2-18 years were diagnosed with disease caused by meningococcal serogroup $C$ in Florida. Over a period of 3 days 13, 148 people received vaccination with a meningococcal ACWY plain polysaccharide vaccine (Menomune ${ }^{\mathrm{TM}}$, Sanofi Pasteur Inc.) to control the outbreak. Vaccine coverage was estimated at $86-99 \%$ at a cost of US $\$ 370,000$. During the year following the campaign, five cases of serogroup $\mathrm{C}$ disease were identified, four of those in patients that had not received the vaccine [69].
The use of digital technologies has the potential to improve communication at the time of a meningococcal disease outbreak [70]. For example, this could be text messages or emails informing school or university students that an outbreak is occurring, and advising them where and when to seek vaccination. There is a risk, however, that the institutionalization and success of such technologies will stand in the way of the alternative approach, which is anticipatory, a priori immunization.

\section{Hajj and other travel-associated disease}

After an outbreak of disease caused by serogroup A during the Hajj pilgrimage in 1987, Saudi Arabia mandated bivalent plain polysaccharide $A$ and $C$ vaccines for pilgrims wishing to enter the country [71]. An outbreak of disease caused by serogroup W-135 occurred during the Hajj in 2000 and 2001 [69]. During subsequent years, outbreaks occurred in Muslim populations in the US, UK, Israel and France [72] as well as in Burkina Faso [110]. The strain responsible for these outbreaks was a serogroup W-135 belonging to the ST-11 complex/ET-37 complex, observed earlier in the UK in serogroup C. In this regard, ST refers to 'sequence type' whilst ET-37 refers to the 'clonal complex', as determined by multilocus sequence typing, a way of subcategorizing sero-groups [no]. This clonality was suggestive of capsular switching [73]. There have been no additional outbreaks in Saudi Arabia since immunization with quadrivalent meningococcal ACWY plain polysaccharide vaccine has been required of all Hajj pilgrims, starting in 2002 [74]. Saudi Arabia and Indonesia switched from plain polysaccharide to quadrivalent conjugate vaccine for Hajj and Umrah pilgrims in 2010.

Meningococcal vaccination, including quadrivalent meningococcal conjugate vaccination, is recommended by many countries for individuals travelling to regions of the world, such as the African meningitis belt and other areas where they are at risk of contracting the disease [21]. Universal meningococcal immunization programs in countries with outbreaks will, at the same time, reduce the risk of travellers to those countries contracting the disease.

\section{Country/region overview}

Epidemiology of meningococcal disease is characterized by its dynamic and unpredictable nature, with great regional variability in disease incidence rates and serogroup distribution. Furthermore, the disease can occur as outbreaks or epidemics, thus highlighting the need for country-specific strategies to vaccine prevention of meningococcal disease.

Meningococcal $\mathrm{C}$ conjugate vaccine has enjoyed universal recommendation and reimbursement in only a small number of countries, including the UK, The Netherlands, Belgium, Spain, Iceland, Ireland, France, Germany, Luxembourg, Portugal, Switzerland, Brazil, Australia and Canada. Italy and Cyprus have at-risk recommendations. Only two countries (the USA and Canada) have had nationwide recommendations and reimbursement of quadrivalent meningococcal conjugate vaccine against serogroups A, C, W-135 and $\mathrm{Y}$, and Greece is now the third. The advantage for policy-makers in recommending a quadrivalent meningococcal conjugate vaccine in countries that may not have the four included serogroups circulating widely is that the recommendation not only anticipates temporal changes in serogroups but also protects travellers 
into and out of the country. Mass vaccination campaigns with a serogroup A conjugate vaccine (MenAfriVac) were introduced in the African meningitis belt countries in 2010. Thus Asia, part of Africa, part of Europe, Russia, the Middle East and most of Latin America rely on outbreak control with chemoprophylaxis, vaccines or both. By contrast, pneumococcal conjugate vaccines are now recommended and reimbursed in many countries $[75,76]$.

In the context of policies and the country/region overview, it is possible that at-risk and universal recommendation policies for serogroup $\mathrm{C}$ meningococcal conjugate vaccine have been driven by an under-awareness, under-diagnosis and under-reporting of meningococcal infection in general and serogroup $C$ infection in particular. This could be due to a perception, for example, that certain countries are 'serogroup C countries whilst others are 'serogroup B' countries. Second, the great success of the 'non blanching rash' test with a glass beaker may have inadvertently sent a (wrong) message that meningococcal infection is thus diagnosable (i.e., make the diagnosis, administer the antibiotics, disease progression averted), whereas the reality is that meningococcal infection may present without the distinctive rash. Third, the policies may have been driven by the 'certainty' that preemptive antibiotics deal with all meningococcal serogroups whilst a serogroup $C$ vaccine only deals with one serogroup. Finally, using the pneumococcal conjugate vaccine 'model', whereby the vaccine covers multiple sero-types, there may have been a delay in implementing meningococcal conjugate vaccine in the expectation of vaccines covering multiple serogroups. Adoption of recommendations of such meningococcal conjugate vaccines into national policies would vindicate to a degree this particular 'more-is-better' hypothesis.

The Asian region deserves special attention. The real burden of disease is not well known with limited information available, due in part to lack of surveillance in many countries. Meningococcal disease in Asia is probably under-recognized, emphasizing the need in improved surveillance for a better understanding of the true burden of disease.

\section{Discussion}

Regardless of national or international meningococcal immunization policies, it is unlikely that global elimination of meningococcal infection can be achieved. In this way, it is similar to Streptococcus pneumoniae, in which there has been considerable success not only in reducing the nasopharyngeal carriage of vaccine serotypes but also in reducing disease as a result of the use of pneumococcal conjugate vaccines [77]. Overt meningococcal and pneumococcal infections can be reduced to verylow levels, but nasopharyngeal carriage of these organisms is more difficult to remove entirely [78]. While serotype replacement has been observed following use of the 7 -valent pneumococcal conjugate vaccine, there is no evidence that this occurs to a clinically significant degree with meningococcal vaccines, although it is possible that meningococcal vaccine policy in some countries has been influenced by a concern that replacement could occur with serogroup $B$ organisms.

In comparing countries with and without universal meningococcal C conjugate programs, the example of France is most salutary. In France, despite peaks of serogroup $\mathrm{C}$ disease in the first decade of this century, a universal recommendation was not forthcoming until 2009. It appears that health economics was an important driver in the delay in implementing universal meningococcal serogroup C immunization in France [29]. Another factor was the realization that by 2007 the rate of invasive meningococcal disease in France was higher than in countries that had already implemented universal meningococcal serogroup C immunization [79].

Regarding adolescent immunization [23], one study highlighted the barriers as being public concerns (e.g., safety), practitioner concerns (e.g., ambivalence about recommendations), delivery issues (e.g., access), consent issues, cost/financing issues and a lack of coordination in timing of vaccine recommendations, supply and financing [24]. This suggests that reassuring the public about the safety profile of meningococcal conjugate vaccines, greater education of practitioners about the scientific rationale for recommendations, improved access to vaccines, a more pragmatic approach to adolescent consent, more research into meningococcal health economics and improved coordination could all result in improvements to adolescent meningococcal vaccination. New policies to enhance adolescent meningococcal vaccination could include 'well visits' to health promotion clinics and education as has occurred with vaccines against the HPV. Although it is likely that adolescents would be motivated towards vaccination themselves for the purpose of providing herd protection amongst their peers, it is less likely that they would be motivated in the same way to provide herd protection to infants and young children.

Contribution of herd protection was of paramount importance to the success of meningococcal $C$ conjugate programs, thus providing an opportunity to bring about a large decrease in disease. The herd protection effect is observed by reductions in the rates of serogroup $\mathrm{C}$ carriage in the population and reductions in serogroup $\mathrm{C}$ disease in unvaccinated individuals. These observations are crucial when deciding the vaccination strategies to be implemented in immunization programs. Catch-up can be performed in any age group either in conjunction with or following an ongoing program. It is not known yet what degree and duration in the reduction in meningococcal nasopharyngeal carriage are required to bring about herd protection [6]. However, a catch-up program adds to the cost of implementation. In Brazil, with an annual birth cohort of 3 million, serogroup $\mathrm{C}$ meningococcal conjugate vaccination was implemented universally in infants and toddlers, but the vaccine was not made available for a catch-up program, despite the expectation of herd immunity protection as seen in other countries where serogroup $C$ vaccine has been introduced with large catchup programs. The decision to include or not include a catch-up program is an important one for policy-makers - they can have a substantial impact on meningococcal disease burden [80].

In the case of sole private market availability of meningococcal conjugate vaccines, when a reliance on herd protection cannot be assumed, infant immunization to confer direct protection is likely to be necessary in addition to vaccination of toddlers, young children and adolescents alone, taking into account the high incidence rates of meningococcal disease consistently reported in the first year of age. The evidence from the UK is that the early herd protection of infants occurred as a result of high serogroup $C$ 
conjugate vaccination rates in adolescents, the most prevalent carriers of meningococci [81]. Whilst there was a national immunization campaign for all those up to 19 years of age, the roll-out to the adolescents occurred early in the campaign. The reduction in disease in the infants started to occur coincident with this, before direct protection of the infants had had time to occur. There is also evidence from Spain of the impact that plain polysaccharide serogroup $C$ meningococcal vaccine has on nasopharyngeal carriage in adolescents, although there are conflicting and inconclusive results [82]. It is also likely that infant vaccinees would need a booster in the future, as older children, adolescents or young adults, to maintain protection. Waning of protection after serogroup $\mathrm{C}$ meningococcal conjugate vaccination at a young age, as recently observed in the Province of Quebec, Canada, is the main justification for including a booster dose during adolescence [50].

Serogroup B OMV meningococcal vaccines have been used in Cuba, where routine vaccination has been in place for 20 years, Norway (as part of a randomized controlled trial) and New Zealand, with an observed decrease in meningococcal serogroup $B$ disease. Although OMV vaccines have proven themselves effective for the control of outbreaks caused by strains homologous with the vaccine, they do not offer protection that covers the remaining meningo-coccus $B$ serosubtypes, in addition to only offering modest immunogenicity in young children. The reactogenicity profile of OMV vaccines may also affect their uptake. To broaden protection, recombinant protein vaccines, one of them containing two recombinant lipidated proteins from the $\mathrm{fHbp}$ subfamilies $\mathrm{A}$ and $\mathrm{B}$, and the other containing neisseria adhesin $A(\operatorname{NadA})$, neisserial heparin-binding antigen (NHBA) and fHbp (subfamily B), in combination with the OMV vaccine from group $B$ strain NZ98/254, were developed, with promising results in clinical trials, albeit immunogenicity rather than efficacy trials, and are likely to become available in the near future [83]. These novel noncapsular protein antigens used for recombinant group B vaccines are shared across strains with other capsular groups, creating the possibility of extending the protection for other serogroup strains. However, the effect of these vaccines on carriage and on protection against non-group B strains in the wild is not known yet, making this information crucial to defining the future strategies to introduce these vaccines. Should meningococcal serogroup $B$ vaccines be found to confer a degree of serogroup/serotype cross-protection, this would be an important and useful quality $[84,85]$. In addition, the need for booster doses of meningococcal serogroup $B$ vaccines to maintain long-term protection is not yet clear. Cost-effectiveness analysis of vaccination programs requires the use of appropriate models that include the indirect effects of vaccination. Pharmacoeconomic considerations do play a part in the decision-making process with regard to meningococcal vaccination and this topic has been covered very well by Welte et al. [27]. These authors described the pharmacoeconomic evaluations that had been performed in six countries, four of which having occurred before the introduction of serogroup C meningococcal conjugate vaccine (Australia, Canada specifically Quebec, The Netherlands and the UK) and two of which having occurred after the introduction (Portugal and Switzerland). However, the authors noted an important principle, namelythat with stronglyincreasingmeningococcal disease incidence, public health considerations took precedence over economic considerations.

In summary, the policy options available for control of meningococcal infection are:

- Routine age-specific recommendations;

- Routine infant strategy plus catch-up (e.g., UK, Spain, Iceland and Portugal) - this approach targets the age group where the incidence is typically the highest and also takes advantage of the high vaccine coverage rate intrinsic to routine infant programs. Countries where a high burden of disease is observed in infants have adopted this strategy;

- Routine toddler strategy plus catch-up (e.g., Australia, The Netherlands, Belgium, France and most of Canada). This schedule was introduced based on epidemiologic and cost-effectiveness analysis, considering the relatively low number of cases of meningococcal disease in infants in these countries. Reductions in incidence rates of disease in infants and also in adults too old to have been vaccinated were most likely the result of the herd protection effect provided by vaccination of adolescents, the age-group primarily responsible for nasopharyngeal carriage;

- Adolescent-onlystrategy(e.g., in the USAand Manitoba)this protects adolescents against the 'second peak' and may provide herd effects/indirect protection;

- Monovalent versus multivalent recommendations;

- To respond to serogroup-specific epidemiology shifts over time and over various geographic regions;

- The use of multivalent vaccines (investigational serogroup A, B, C, W135, Y or other serogroup combinations) even where one or more of the included serogroups are uncommon/nonexistent, to provide the broadest coverage possible.

\section{Expert commentary}

Meningococcal disease continues to be a serious public health threat. The disease is often sudden in onset, rapidly progressive and associated with high case-fatality rates even after treatment. Serogroup distribution and disease incidence varies globally, regionally and over time. Because of this dynamic nature of the meningococcus epidemiology, vaccination policies should have countryspecific recommendations and be reviewed regularly in order to be aligned with epidemiology and changing disease patterns.

Meningococcal $\mathrm{C}$ conjugate vaccines proved to be highly effective, with a dramatic reduction in the incidence of meningococcal disease caused by serogroup $C$ in countries that have introduced them into their mass immunization programs. Reductions in serogroup $\mathrm{C}$ disease occurred not only in vaccinated but also in unvaccinated individuals, indicating the presence of herd immunity.

Long-term effectiveness of meningococcal conjugate vaccines is dependent on a combination of persistence of antibodies, immunologic memory and herd protection. Evidence indicating that antibody persistence is poor after immunization in infants and immunologic memory is not fast enough to protect them against immediate disease, emphasizes the importance of herd immunity in providing sustained indirect protection [40]. For example, infants younger than the age of first vaccination are susceptible 
to disease and infants between the first and the second vaccination may also be susceptible. However, herd immunity is expected to diminish with time as these susceptible cohorts become potentially transmitting adolescents and adults. This way, the introduction of a booster dose during adolescence for those children immunized before school age is likely to be necessary and should be considered to maintain a population protected against the disease. Using the quadrivalent $\mathrm{A}, \mathrm{C}, \mathrm{W}-135$ and $\mathrm{Y}$ meningococcal conjugate vaccine as the booster dose would provide a wider protection, acting as a booster for serogroup $C$ and as a priming dose for serogroups $\mathrm{A}, \mathrm{Y}$ and $\mathrm{W}-135$.

Licensed meningococcal conjugate vaccines and the investigational meningococcal $B$ vaccines, once they are licensed, have the potential to reduce the incidence of invasive meningococcal disease to very low levels. Robust evaluation of the meningococcal $B$ vaccines either by postlicensure effectiveness studies, surveillance studies or both, would confirm or refute that potential. Universal programs are preferable to programs that target those deemed to be highly at risk, those who find themselves in the midst of an outbreak or travelers, because it is difficult to identify and vaccinate all those at risk for such reasons. The potential for sustained reductions in disease arising from fewer doses of meningococcal vaccine administered in infancy, a greater reliance on herd protection of infants through adolescent vaccination and inclusion of an adolescent booster dose for those infants when they reach adolescence should be explored in long-term studies.

\section{Five-year view}

An increase of serogroup $\mathrm{Y}$ disease, caused by clone $\mathrm{Yl}$, has recently been observed in northern European countries [86]. A small but marked increase in serogroup $Y$ disease has also been reported in the UK [in]. It is possible that this serogroup will gain in importance.

In addition to environmental and treatment aspects, it is possible that certain host protective factors may play a part in the reporting of disease [87]. Detailed seroepidemiology and genetic studies may elucidate further the role of host protective factors.

In the future we will most likely see the increased use of meningococcal conjugate vaccines instead of polysaccharide vaccines, whenever possible. The increasing use of both quadrivalent serogroup A, C, W-135, $Y$ meningococcal conjugate vaccines (MenACWY-D and MenACWY-CRM) and other multivalent conjugate vaccines currently at the investigational stage, such as MenACWY-TT and the combination Haemophilus influenza type b plus $N$. meningitidis serogroups $C$ and $Y$ conjugate vaccine will begin to provide the necessary data to evaluate the impact of the vaccines on the carriage of serogroups $A$, W-135 and $\mathrm{Y}$, and the potential for herd immunity and indirect protection. The licensure and deployment of the currently investigational recombinant protein vaccines against serogroup $B$ disease, combined with more robust evaluation of their reac-togenicity and immunogenicity profiles, particularly in infants, will determine their impact on the acquisition of carnage, their effectiveness and the coverage against group B and non-group B strains, since the protein antigens used in these vaccines are shared with other serogroup strains. This information will be crucial to determine which age groups should be vaccinated. Improvements to current immunization schedules, especially addressing both infant and adolescent immunization and general coordination of schedules will most likely occur. The possibility of maternal immunization is likely to be explored as another option for protecting young infants.

\section{Acknowledgements}

The authors would like to thank Lisa De Tora, Michael Broeker and Jeff Stoddard for their critical reading of the manuscript and comments.

\section{Financial \& competing interests disclosure}

MAP Safadi has received consultation and lecture fees from Baxter, GlaxoSmithKline, MSD, Novartis, SanofiPasteur and Wyeth. EDG Mclntosh is an employee of Novartis Vaccines and Diagnostics. The authors have no other relevant affiliations or financial involvement with any organization or entity with a financial interest in or financial conflict with the subject matter or materials discussed in the manuscript apart from those disclosed.

Writing assistance was utilized in the production of this manuscript. The authors would like to acknowledge $K R$ Collins from JK Associates (Conshohocken, PA, USA) for editorial assistance and manuscript preparation funded by Novartis Vaccines and Diagnostics.

Meningococcal disease is characterized by a variation in incidence and serogroup distribution by region and temporally.

Routine immunization programs targeting meningococcal disease caused by serogroup $C$ have been highly effective at disease reduction. A reduction of serogroup $C$ disease has occurred in both vaccinated and unvaccinated individuals, indicating herd protection.

Recent data indicate that long-term effectiveness of meningococcal conjugate vaccines may be inadequate and a booster dose may be required in adolescence to maintain protection.

The role for multivalent meningococcal conjugate vaccines in outbreak control and risk programs is likely to increase; their role in replacing monovalent meningococcal $C$ conjugate vaccine is less clear.

Investigational serogroup $B$ vaccines will most likely play a role in the further reduction of invasive meningococcal disease. 